\title{
$V_{u b}$ AND WEAK ANNIHILATION IN INCLUSIVE SEMILEPTONIC $D / D_{s}$ DECAYS
}

\author{
JERNEJ F. KAMENIK \\ J. Stefan Institute, Jamova 39, P. O. Box 3000 \\ 1001 Ljubljana, Slovenia \\ jernej.kamenik@ijs.si
}

\begin{abstract}
We search for signals of Weak Annihilation in inclusive semileptonic $D$ decays. We consider both the widths and the lepton energy moments. The analysis of Cleo data shows no clear evidence of Weak Annihilation, and allows us to put bounds on its relevance in charmless $B$ semileptonic decays.

Keywords: D semileptonic decays; B semileptonic decays and Vub determination; Weak Annihilation; Heavy quark expansion.
\end{abstract}

PACS numbers: 13.20.Fc, 12.39.Hg, 12.15.Hh

\section{Introduction}

The value of $\left|V_{u b}\right|$ preferred by current global analyses of CKM data is about $15 \%$ smaller than the one extracted from inclusive charmless semileptonic $B$ decays ${ }^{1}$. Though not very significant, the discrepancy has prompted a reexamination of the sources of theoretical uncertainty in the inclusive determination ${ }^{2}$. Weak Annihilation (WA) contributions are generally considered an important source of uncertainty in the Operator Product Expansion (OPE) that describes the inclusive $B$ decays ${ }^{3}$, and affect especially the high $q^{2}$ and lepton endpoint analyses. They appear in the OPE as $1 / m_{b}^{3}$ corrections involving the matrix elements of dimension- 6 four-quark operators, and affect both the total $B \rightarrow X_{u} \ell \bar{\nu}$ decay rate and the charged lepton energy spectrum ${ }^{4,5}$.

It was noted in refs. 6,7 that the WA matrix elements that enter $B \rightarrow X_{u} \ell \bar{\nu}$ decay can be constrained via the semileptonic decays of $D$ and $D_{s}$ mesons, using heavy quark symmetry. Several authors have attempted to extract information on WA contributions from the measured total semileptonic rates of $D^{0, \pm}$ and $D_{s}^{8,9,10}$. For instance, one may attribute the observed differences in $D^{ \pm, 0}$ and $D_{s}$ semileptonic widths ${ }^{11}$ to the valence spectator quark WA contributions in $D_{s}$ decays, since they are Cabibbo suppressed in the $D^{ \pm}$case and completely absent in $D^{0}$ decays. However, additional contributions to this difference arise from $\mathrm{SU}(3)$ breaking in 
the matrix elements of all dimension 5 and 6 operators, that contribute significantly to the total rates ${ }^{6}$.

A complementary strategy is to consider also the moments of the lepton energy spectra in the OPE and compare them with recent experimental results from Cleo ${ }^{11}$. Not only are the moments free of the strong dependence on the charm quark mass and its associated uncertainty, but their perturbative and non-perturbative corrections tend to cancel as well. Moreover, since WA is expected to dominate the spectrum endpoint, leptonic moments might be more sensitive to WA contributions than the total rate.

\section{Experimental data}

Recently the Cleo Collaboration has measured the electron spectra of inclusive semileptonic $D^{ \pm, 0}$ and also $D_{s}$ decays ${ }^{11}$ with a lower cut on the electron momentum in the lab frame of $p_{e}>0.2 \mathrm{GeV}$. They extract the total decay rates by extrapolating the spectra over the remaining phase-space, using a theoretically modeled sum over known exclusive modes. Unfortunately, they do not provide higher leptonic energy moments.

In Ref. 12 the moments without cuts have been extracted by first extrapolating the measured spectra towards $p_{e}=0$ using OPE inspired extrapolation formulae and then boosting the results from the laboratory to the $D$ rest-frame. Within the stated uncertainties there is no sign of a difference between the moments of $D_{s}$ and $D^{0, \pm}$. This is at odds with what one would naively expect from what is observed in the rates ${ }^{11}$. However, as mentioned in the Introduction, the concurring contribution of $\mathrm{SU}(3)$ violation in the matrix elements of higher dimensional operators might provide a partial explanation. In order to reliably extract possible WA contributions from the measured total semileptonic rates of $D$ and $D_{s}$ mesons ${ }^{11}$, one would therefore need to estimate the size of $S U(3)$ violation in all these matrix elements. In the case of normalized moments, on the other hand, some of the leading power corrections cancel out and one might be more directly sensitive to WA contributions.

\section{Inclusive semileptonic charm decays in the OPE}

We have calculated the semileptonic rate and the total moments from the lepton energy spectra in terms of $x \equiv 2 E_{e} / m_{c}$, where $E_{e}$ is the electron energy in the $D$ meson inertial frame. For simplicity, we have only considered the total leptonic moments, without lower cuts on the lepton energy, and have neglected the lepton mass as well as Cabibbo suppressed contributions. The unnormalized leptonic energy moments are defined as

$$
\Gamma^{(n)} \equiv \int_{0}^{(1-r)} \frac{d \Gamma}{d x} x^{n} d x,
$$


where $r \equiv\left(m_{s} / m_{c}\right)^{2}$. The WA contributions to to the $n$-moment appear at the order $1 / m_{c}^{3}$

$$
\Gamma_{\mathrm{WA}}^{(n)}=\frac{G_{F}^{2} m_{c}^{5}}{192 \pi^{3}}\left|V_{c s}\right|^{2}\left[\frac{32 \pi^{2}}{m_{c}^{3}} B_{\mathrm{WA}}^{(n)}\right] .
$$

The $B_{\mathrm{WA}}^{(n)}$ can be identified with the matrix elements of the dimension- 6 four quark operators $\left(\mathcal{O}_{1,2}^{Q q}\right.$, where $Q$ and $q$ are the heavy and light quark flavor indices respectively) entering charm meson decay rates

$$
B_{\mathrm{WA}}^{c q}\left(\mu_{\mathrm{wA}}\right)=\frac{1}{2 m_{D}}\left\langle D\left|\mathcal{O}_{2}^{c q}-\mathcal{O}_{1}^{c q}\right| D\right\rangle=\frac{1}{2} m_{D} f_{D}^{2}\left(B_{D, 2}^{q}-B_{D, 1}^{q}\right),
$$

where $B_{D, i}^{q}\left(\mu_{\mathrm{wA}}\right)$ parameterize the deviation from the factorization approximation at the renormalization scale $\mu_{\mathrm{WA}}: B_{\mathrm{WA}}^{Q q}$ vanishes in the limit of factorization.

Neglecting small Cabibbo suppressed and isospin-violating effects, the WA contributions to the decays of charmed mesons involving different spectator quarks can be described in terms of just two distinct contributions, which can be identified with the valence and non-valence WA terms involving the $s$ quark. The first one, $B_{\mathrm{WA}}^{c s}\left(D_{s}\right)$, only contributes to the $D_{s}$ decays, while the second one, $B_{\mathrm{WA}}^{c s}(D)$, contributes equally to $D^{+}$and $D^{0}$ decays. In the flavor $\mathrm{SU}(3)$ limit, the two contributions correspond to the sum of isotriplet $\left(\Delta B_{\mathrm{WA}}^{c}\right)$ and isosinglet $\left(B_{\mathrm{WA}}^{c}\right)$, and to the isosinglet contribution, respectively.

As it was recognized long ago ${ }^{4}$, WA is localized at the endpoint of the lepton energy spectrum, and can be approximately expressed by a delta function at the partonic endpoint. In this case, we would expect $B_{\mathrm{WA}}^{(n)}=B_{\mathrm{WA}}^{c s}$, for all $n$, up to small $\mathcal{O}(r)$ effects. In fact, gluon bremsstrahlung and hadronization effects are expected to smear the WA contribution over a region of electron energy around the partonic threshold $\left(m_{c}^{2}-m_{s}^{2}\right) / 2 m_{c}$. The size and shape of the smearing may affect the various integrals $B_{\mathrm{WA}}^{(n)}$ differently and cannot be predicted, although one expects a small perturbative tail to emerge away from the endpoint. Indeed, the WA distributions spread over a region of electron energies of $\mathcal{O}\left(\Lambda_{Q C D}\right)$. We are primarily interested in a determination of the leading matrix element, $B_{\mathrm{WA}}^{(0)}$, i.e. the zeroth moment of the WA distribution, from $B_{\mathrm{WA}}^{(n)}$ : the non-negligible smearing of WA may lead to a model-dependent dilution. In order to quantify this effect on the parameters $B_{\mathrm{WA}}^{(n)}$, we have considered a number of smearing distributions ${ }^{12}$ and found that in general the dilution is expected to be a moderate $\mathcal{O}(20 \%)$ effect.

\section{Results and Discussion}

First we have checked whether the OPE at $\mathcal{O}\left(1 / m_{c}^{3}\right)$ describes the experimental data in a satisfactory way. Working in the pole mass scheme, the lepton moments receive smaller perturbative corrections than the total rate, while they are very sensitive to WA and the Darwin term $\rho_{D}^{3}$ also contributing at $1 / m_{c}^{3}$. As for the $m_{c}$ dependence, since $\left\langle E^{n}\right\rangle$ scales like $m_{c}^{n}$, the lowest moments are less sensitive to the value of the charm mass than the width, which is proportional to $m_{c}^{5}$. 
In order to avoid the well-known problems associated to the pole mass, semileptonic decays are usually described in terms of threshold heavy quark masses, like the kinetic mass, the PS, or the $1 \mathrm{~S}$ mass, see Ref. 2 for a review. Working in the kinetic scheme ${ }^{13}$, the dominant source of parametric uncertainty in the first and the second leptonic energy moment is by far $\rho_{D}^{3}$, while in the total rate the charm quark mass uncertainty dominates. Varying all the input parameters within conservative ranges we have extracted the values of $B_{\mathrm{wA}}^{(0,1,2)}\left(D_{q}\right)$ by comparing the theoretical predictions with experimental rates and moments ${ }^{12}$. At the estimated theoretical precision, all the extracted WA contributions are consistent with zero. The obvious implication is that the OPE describes all the data reasonably well.

As already mentioned in the previous section, we expect WA to be concentrated at the end-point, in which case we can combine the $D^{0,+}$ results to obtain the isosinglet $B_{\mathrm{WA}}^{(0)}\left(\mu_{\mathrm{WA}}=0.8 \mathrm{GeV}\right)=-0.0003(15) \mathrm{GeV}^{3}$. In the worst case, the reduced sensitivity due to the WA smearing functions is about $60 \%$, which leads to

$$
B_{\mathrm{WA}}^{c}\left(\mu_{\mathrm{WA}}=0.8 \mathrm{GeV}\right)=-0.0003(25) \mathrm{GeV}^{3},
$$

where we have combined the dilution error linearly, without changing the central value. We regard this as sufficiently conservative.

We can also look for indications of WA dilution by extracting $B_{\mathrm{WA}}^{(0,1,2)}$ from a combined fit to the moments and the total rates. The results for $D^{0}$ and $D_{s}$ decays are shown in figure 3 of Ref. 12 and are consistent with $B_{\mathrm{WA}}^{(0)} \approx B_{\mathrm{WA}}^{(1)} \approx B_{\mathrm{WA}}^{(2)}$, but the errors are too large to draw a conclusion concerning WA dilution.

In order to connect to $B \rightarrow X_{u} \ell \nu$ we refer to the WA matrix elements determined in charm decays as $B_{\mathrm{WA}}^{c}$ and consider their relation to those relevant in $B$ semileptonic decays, $B_{\mathrm{WA}}^{b}$, taking into account eq. (3). In the heavy quark limit ${ }^{14}$, $f_{P} \sim m_{P}^{-1 / 2}$ so that $B_{P, i}$ scale as constants with heavy quark mass, but recent lattice results give ${ }^{15} f_{D} \approx 0.21 \mathrm{GeV}, f_{B} \approx 0.20 \mathrm{GeV}$. We also neglect any evolution of the WA operators and write

$$
B_{\mathrm{WA}}^{b}\left(\mu_{\mathrm{WA}}\right)=\frac{m_{B} f_{B}^{2}}{m_{D} f_{D}^{2}} B_{\mathrm{WA}}^{c}\left(\mu_{\mathrm{WA}}\right) .
$$

The parametric enhancement due to meson masses and decay constants is a significant factor of 2.5. From eqs. $(4,5)$ we derive a bound

$$
\left|B_{\mathrm{WA}}^{b}\left(\mu_{\mathrm{WA}}=0.8 \mathrm{GeV}\right)\right| \lesssim 0.006 \mathrm{GeV}^{3},
$$

which holds for the non-valence contributions, although we stress that the data seem to prefer even smaller values. The valence contribution is more constrained from the ratio of the $D_{s}$ and $D^{0}$ rates and leptonic moments, from which we expect

$$
-0.004 \mathrm{GeV}^{3} \lesssim \Delta B_{\mathrm{WA}}^{b} \lesssim 0.002 \mathrm{GeV}^{3}
$$

again at $\mu_{\mathrm{WA}}=0.8 \mathrm{GeV}$. The bounds lead to a maximum $2 \%$ WA correction to the total rate of $B \rightarrow X_{u} \ell \nu$. In turn, this translates into an uncertainty of $1 \%$ on $\left|V_{u b}\right|$ extracted from the total rate and from the most inclusive experimental analyses, like those that involve a lower cut on the invariant hadronic mass. 


\section{Summary}

Recent analyses ${ }^{10,12}$ have demonstrated that the heavy quark expansion can be employed to describe inclusive semileptonic $D$ decays and to constrain WA effects in the extraction of $\left|V_{u b}\right|$. In addition to the total widths, the Cleo data on the

lepton energy spectra have been used to compute the first few moments ${ }^{12}$. The latter are quite sensitive probes of possible WA contributions, both in its isosinglet and isotriplet components, and determine very precisely a linear combination of the expectation values of the Darwin and WA operators.

The analysis of Cleo data shows no clear evidence for WA, i.e. the OPE describes well the experimental results even in the absence of WA. An upper limit on both valence and non-valence WA components can be derived, which allows to put a bound of $2 \%$ on their relevance in the $B \rightarrow X_{u} \ell \nu$ decay rate and even less for the isotriplet component.

\section{Acknowledgments}

The author would like to thank Paolo Gambino for a fruitful collaboration as well as the organizers of CHARM 2010 for the invitation to this exciting conference. This work is supported in part by the European Commission RTN network, Contract No. MRTN-CT-2006-035482 (FLAVIAnet) and by the Slovenian Research Agency.

\section{References}

1. M. Bona et al. [UTfit Collaboration], Phys. Lett. B687, 61-69 (2010), for recent $\left|V_{u b}\right|$ averages see http://www.slac.stanford.edu/xorg/hfag/.

2. M. Antonelli et al., Phys. Rept. 494, 197-414 (2010).

3. J. Chay, H. Georgi, and B. Grinstein, Phys. Lett. B247 (1990) 399; I. I. Y. Bigi, N. G. Uraltsev, and A. I. Vainshtein, Phys. Lett. B293 (1992) 430; I. I. Y. Bigi, M. A. Shifman, N. G. Uraltsev, and A. I. Vainshtein, Phys. Rev. Lett. 71 (1993) 496; A. V. Manohar and M. B. Wise, Phys. Rev. D49 (1994) 1310.

4. I. I. Y. Bigi and N. G. Uraltsev, Nucl. Phys. B 423, 33 (1994).

5. R. D. Dikeman and N. G. Uraltsev, Nucl. Phys. B 509, 378 (1998); I. I. Y. Bigi, R. D. Dikeman and N. Uraltsev, Eur. Phys. J. C 4, 453 (1998).

6. I. I. Y. Bigi and N. G. Uraltsev, Z. Phys. C 62, 623 (1994).

7. N. Uraltsev, Int. J. Mod. Phys. A 14 (1999) 4641; M. B. Voloshin, Phys. Lett. B 515, $74(2001)$.

8. D. Becirevic, S. Fajfer and J. F. Kamenik, Phys. Lett. B 671, 66 (2009).

9. I. Bigi, T. Mannel, S. Turczyk and N. Uraltsev, JHEP 1004, 073 (2010).

10. Z. Ligeti, M. Luke and A. V. Manohar, Phys. Rev. D82, 033003 (2010).

11. D. M. Asner et al. [The CLEO Collaboration], Phys. Rev. D81, 052007 (2010).

12. P. Gambino and J. F. Kamenik, Nucl. Phys. B840, 424-437 (2010).

13. I. I. Y. Bigi, M. A. Shifman, N. Uraltsev and A. I. Vainshtein, Phys. Rev. D 56 (1997) 4017. and Phys. Rev. D 52 (1995) 196.

14. B. Grinstein, E. E. Jenkins, A. V. Manohar, M. J. Savage and M. B. Wise, Nucl. Phys. B 380, 369 (1992).

15. V. Lubicz and C. Tarantino, Nuovo Cim. 123B, 674 (2008). 